\title{
A NOTE ON NON-AFFINE SOLUTIONS OF TERM STRUCTURE EQUATIONS WITH APPLICATIONS TO POWER EXCHANGES
}

\author{
JOSEF TEICHMANN
}

\begin{abstract}
A method based on transformations of well-known solutions of term structure equations is presented in order to incorporate Martin Barlow's spot price model for electricity into a model for future prices on electricity. The setting for the evolution of term structures is chosen in the spirit of $\mathrm{Da}$ Prato-Zabczyk.
\end{abstract}

\section{INTRODUCTION}

The purpose of this small note is to unify two well-known approaches to model features in electricity markets: the spot price model of Martin Barlow (see [1] for all details), which is an ad hoc micro-economic model reflecting some particular features of electricity exchanges such as price peaks, and a general affine model for the term structure of future or forward prices (see [2] for all fundamentals) on commodities. The idea is to incorporate more flexibly the market anomalies for spot prices such as short-selling constraints or storage costs.

We have tried to incorporate these anomalies through weakening of the assumptions on models of futures market. Similar reasonings hold for forward markets, or for term structure problems in general. In the introduction we present the microeconomic model of Martin Barlow and a general framework for models on future prices, where market anomalies can be represented.

1.1. Martin Barlow's microeconomic model. Martin Barlow's model for power spot prices is derived via the following microeconomic consideration: depending on current prices $x$, there are given a time-dependent, random demand function $d_{t}(x)$ and a time-dependent, random supply function $u_{t}(x)$. Assume that $x \mapsto u_{t}(x)$ is increasing and $x \mapsto d_{t}(x)$ is decreasing and that there is a (unique) price $S_{t}$ for each time point $t$ given through the basic equation

$$
u_{t}\left(S_{t}\right)=d_{t}\left(S_{t}\right)
$$

The assumptions on $u$ and $d$ in [1] are amazingly simple: Supply $u_{t}$ is supposed to be non-random and independent of time $t$

$$
u_{t}(x)=u(x),
$$

demand $d_{t}$ is supposed to be inelastic, but randomly time dependent

$$
d_{t}(x)=D_{t} .
$$

Josef Teichmann gratefully acknowledges the support by the Research Training Network HPRN-CT-2002-00281. 
This yields the nice formula $S_{t}=u^{-1}\left(D_{t}\right) \wedge M$, where $M$ is some ad hoc boundary value for prices. Taking $u(x)=a_{0}+b_{0} x^{c_{0}}$ we obtain a model for the spot price (with some additional boundary assumptions) for either $b_{0}, c_{0}>0$ or $b_{0}, c_{0}<0$,

$$
S_{t}=\left\{\begin{array}{c}
\left(\frac{D_{t}-a_{0}}{b_{0}}\right)^{\frac{1}{c_{0}}} \text { for } D_{t}<a_{0}-b_{0} \epsilon_{0} \\
\epsilon_{0}^{\frac{1}{c_{0}}} \text { for } D_{t} \geq a_{0}-b_{0} \epsilon_{0} .
\end{array}\right.
$$

for $t \geq 0$ and appropriate $\epsilon_{0}$. More precisely: given an Ornstein-Uhlenbeck process $X_{t}$ with $d X_{t}=-\lambda\left(X_{t}-a\right) d t+\sigma d W_{t}$ and $X_{0} \in \mathbb{R}$, we define with $f_{\alpha}(x)=(1+\alpha x)^{\frac{1}{\alpha}}$

$$
S_{t}=\left\{\begin{array}{c}
f_{\alpha}\left(X_{t}\right) \text { for } 1+\alpha X_{t}>\epsilon_{0} \\
\epsilon_{0}^{\frac{1}{\alpha}} \text { for } 1+\alpha X_{t} \leq \epsilon_{0}
\end{array}\right.
$$

for $t \geq 0$ and some $\epsilon_{0}>0$. This model is statistically (with respect to the real world measure) much more satisfying than other spot price models. Values for $\alpha$ like 0.19 , -1.08 or -0.35 are attained for concrete markets explaining very well the particular spot price behaviours. Values like 0 or 1 , which correspond to "classical" spot price models are tested to be non-adequate (see [1] for all details). In our approach we shall consider a smoothened version of $f_{\alpha}$ at the upper boundary value in order to work with nice Ito processes.

1.2. Framework for Term structure of Future Prices. Basic Theory on forwards, futures and bonds can be found in the excellent article [2] and in [3]. For the remainder of this note we assume zero interest rate. Future prices $F(t, T)$ are prices of traded contracts at time $t$ for the delivery of the commodity at maturity time $T$. We do not take all assumptions from [2], we only assume that markets are ideal up to some critical time value $\delta$ before maturity time $T$. Given continuous semi-martingales $(F(t, T))_{0 \leq t \leq T}$ representing future prices with delivery date $T$ at time $t$ on a commodity $\left(S_{t}\right)_{t \geq 0}$, we assume that

: for all delivery times $T \geq 0$ future-prices are given.

: there is $\delta \geq 0$ such that for $T \geq \delta$ the market of futures is ideal (short-selling allowed, liquid) up to time $T-\delta$.

By no-arbitrage arguments we know that $F(T, T)=S_{T}$ for $T \geq 0$. Furthermore we assume

: that there exists an locally equivalent measure $Q$ with the property that semi-martingales $(F(t, T))_{0 \leq t \leq T-\delta}$ are local $Q$-martingales for $T \geq \delta$.

In particular we obtain

$$
F(t, T)=E_{Q}\left(F(T-\delta, T) \mid \mathcal{F}_{t}\right)
$$

for $T \geq \delta$ and $0 \leq t \leq T-\delta$. The formula $E_{Q}\left(S_{T} \mid \mathcal{F}_{t}\right)=F(t, T)$ for $0 \leq t \leq T$ therefore only holds approximately, depending on the choice of $\delta$ and the dynamic of $(F(t, T))_{0 \leq t \leq T}$.

We represent the future prices by

$$
F(t, T)=\exp (\phi(t, T))
$$

assuming in the sequel that they are positive. We can equally describe models for $(F(t, T))_{0 \leq t \leq T}$ or for $(\phi(t, T))_{0 \leq t \leq T}$. This is not the usual representation, which is 
given through

$$
F(t, T)=S_{t} \exp \left(\int_{t}^{T}(f(t, s)-c(t, s)) d s\right)
$$

with forward rates $(f(t, T))_{0 \leq t \leq T}$ and convenience yields $(c(t, T))_{0 \leq t \leq T}$ (see for instance [2]). We have the relation

$$
\phi(t, T)=\ln S_{t}+\int_{t}^{T}(f(t, s)-c(t, s)) d s .
$$

In particular we have $\phi(T, T)=\ln S_{T}$ for $T \geq 0$.

Additionally we shall specify a framework for models with respect to a given $d$-dimensional Brownian motion $\left(W_{t}\right)_{t \geq 0}$. Under the above assumptions we can formally derive stochastic PDEs for the future prices

$$
d F(t, T)=\alpha(t, T) d t+\sum_{i=1}^{d} \beta_{i}(t, T) d W_{t}^{i}
$$

on a stochastic basis $(\Omega, \mathcal{F}, Q)$ with a $d$-dimensional Brownian motion $\left(W_{t}\right)_{t \geq 0}$, where $Q$ denotes the locally equivalent martingale measure. Notice that it follows from 1.2 that $\alpha(t, T)=0$ if $T-t \geq \delta$. The Musiela parametrization $x=T-t$ is applied and we obtain for $\phi$ the following SPDE

$$
d \phi(t, x)=\left(\frac{d}{d x} \phi(t, x)-\frac{1}{2} \sum_{i=1}^{d} \sigma_{i}^{2}(t, x)+\gamma(t, x)\right) d t+\sum_{i=1}^{d} \sigma_{i}(t, x) d W_{t}^{i}
$$

where $\gamma(t, x)=0$ if $x \geq \delta$ in the martingale measure $Q$. In particular the evaluation of $\phi$ at $x=0$ yields the spot price dynamics with respect to $Q$. Solutions of this equation for initial term structures of future prices provide solutions for the stochastic evolution of future prices in the martingale measure (for a more detailed exposition see [5]).

In classical term structure models always $\gamma(t, x)=0$ holds for $t, x \geq 0$. This yields in particular that the spot prices $S_{t}$ are geometric Brownian motions or geometric Bessel processes such as in interest rate theory. The term structure of future prices is then affine (see also [6]).

It is the goal of this article to show that the introduction of the drift $\gamma$ leads to models, where the spot price is given through the microeconomic model 1.1, and the term structure of future prices is affine for $x \geq \delta$. In particular for $0 \leq x \leq \delta$ we therefore have non-affine term structures.

\section{NON-AFFine TERM STRUCTURES}

In this section we provide a nice class of examples for equation 1.5 , which are analytically tractable and which incorporate the microeconomic spot price behaviour.

We take the usual assumption on the stochastic basis $(\Omega, \mathcal{F}, Q)$, the filtration $\left(\mathcal{F}_{t}\right)_{t \geq 0}$ and the $d$-dimensional Brownian motion $\left(W_{t}^{1}, \ldots, W_{t}^{d}\right)_{t \geq 0}$. In our setting a square-integrable Ito process is given through

$$
X_{t}=X_{0}+\int_{0}^{t} b_{s} d s+\sum_{i=1}^{d} \int_{0}^{d} a_{s}^{i} d W_{s}^{i}
$$

with $b$ integrable and $a^{i}$ square-integrable, progressively measurable $H$-valued processes. Ito's formula reads as follows, see [4], chapter 4, for details: 
Theorem 1. Suppose $X$ is an $H$-valued Ito process and $\psi \in C_{b}^{2}(H ; H)$. Then $D \psi\left(X_{t}\right)\left(a_{t}^{i}\right)$ and

$$
J_{t}:=\frac{1}{2} \sum_{i=1}^{d} D^{2} \psi\left(X_{t}\right)\left(a_{t}^{i}, a_{t}^{i}\right)
$$

satisfy the square-integrability conditions for $H$-valued Ito integrals and

$$
\begin{aligned}
(\psi \circ X)_{t} & =\psi\left(X_{0}\right)+\int_{0}^{t}\left(D \psi\left(X_{s}\right)\left(b_{s}\right)+J_{s}\right) d s+ \\
& +\sum_{i=1}^{d} \int_{0}^{t} D \psi\left(X_{s}\right)\left(a_{s}^{i}\right) d W_{s}^{i} .
\end{aligned}
$$

This theorem also has a local version for $\psi \in C^{2}(H ; H)$.

For the general theory we investigate the following equation

$$
d \phi_{t}=\left(A \phi_{t}+\alpha\left(\phi_{t}\right)\right) d t+\sum_{i=1}^{d} \beta_{i}\left(\phi_{t}\right) d W_{t}^{i},
$$

where $\alpha, \beta_{1}, \ldots, \beta_{d}: U \rightarrow H$ are locally Lipschitz vector fields on the open subset $U \subset H$ and $A$ is the generator of a strongly continuous semigroup $\left(T_{t}\right)_{t \geq 0}$ on $H$.

Solutions of equation 2.1 are defined in a subtle way. A strong, continuous solution of 2.1 with initial value $\phi^{*}$ is a stochastic process with continuous paths $\left(\phi_{t}\right)_{t \geq 0}$ together with a strictly positive stopping time $\tau$ such that $\phi_{t} \in \mathcal{D}(A)$, $\left(A \phi_{t}\right)_{t \geq 0}$ for $0 \leq t \leq \tau$ and

$$
\phi_{t}=\phi^{*}+\int_{0}^{t} A \phi_{s} d s+\int_{0}^{t} \alpha\left(\phi_{s}\right) d s+\sum_{i=1}^{d} \int_{0}^{t} \beta_{i}\left(\phi_{s}\right) d W_{s}^{i}
$$

for $0 \leq t \leq \tau$.

A mild, continuous solution of 2.1 with initial value $\phi^{*}$ is a stochastic process with continuous paths $\left(\phi_{t}\right)_{t \geq 0}$ together with a strictly positive stopping time $\tau$ such that

$$
\phi_{t}=T_{t} \phi^{*}+\int_{0}^{t} T_{t-s} \alpha\left(\phi_{s}\right) d s+\sum_{i=1}^{d} \int_{0}^{t} T_{t-s} \beta_{i}\left(\phi_{s}\right) d W_{s}^{i}
$$

for $0 \leq t \leq \tau$. Clearly every strong, continuous solution is a mild, continuous solution by variation of constants (see [4]).

Proposition 1. For any initial value $\phi^{*} \in U$ there is a unique local mild, continuous solution of equation 2.1 under the stated Lipschitz conditions on $\alpha, \beta_{1}, \ldots, \beta_{d}$. Given $\phi^{*} \in U$, we can choose an open neighborhood $W$ of $\phi^{*}$ in $U$ and a stopping time $\tau$, such that there exist mild, continuous solutions $\left(\eta_{t}\right)_{t \geq 0}$ with initial value $\eta_{0} \in W$ and associated stopping time $\tau$. Furthermore, for any sequence $\left(\eta_{0}^{n}\right)_{n \geq 1}$ in $W$ with $\eta_{0}^{n} \rightarrow \eta_{0}$ in $H$ the solutions $\left(\eta_{t}^{n}\right)_{t \geq 0}$ of equation 2.1 with initial value $\eta_{0}^{n}$ satisfy

as $n \rightarrow \infty$.

$$
E\left(\sup _{0 \leq t \leq \tau}\left\|\eta_{t}^{n}-\eta_{t}\right\|_{H}^{2}\right) \rightarrow 0
$$

Proof. For the proof and the notions of uniqueness, also for stochastic flows on Hilbert spaces, see [4], chapter 7 . 
We introduce the Hilbert spaces $\mathcal{D}\left(A^{k}\right)$ by recursion for $k \geq 0$,

$$
\mathcal{D}\left(A^{k}\right):=\left\{\phi \in \mathcal{D}(A) \text { such that } A \phi \in \mathcal{D}\left(A^{k-1}\right)\right\} .
$$

The scalar products are given by

$$
\left\langle\phi_{1}, \phi_{2}\right\rangle_{\mathcal{D}\left(A^{k}\right)}:=\sum_{i=0}^{k}\left\langle A^{i} \phi_{1}, A^{i} \phi_{2}\right\rangle
$$

for $\phi_{1}, \phi_{2} \in \mathcal{D}\left(A^{k}\right)$.

Proposition 2. Assume that $\alpha, \beta_{1}, \ldots, \beta_{d}$ are locally Lipschitz from $(H,\langle.,\rangle$.$) to$ $\left(\mathcal{D}(A),\langle., .\rangle_{\mathcal{D}(A)}\right)$, then every mild, continuous solution of 2.1 with initial value $\phi^{*} \in$ $\mathcal{D}(A)$ is a strong, continuous solution of 2.1 .

Proof. This is a consequence of Proposition 6.4 in [4]. We can also define and solve the equation on $\mathcal{D}(A)$ as a Hilbert space.

Transformations under diffeomorphisms apply the notion of push forward of vector fields, i.e. let $X$ be a vector field on $U \subset H$ and $\psi: U \rightarrow V$ be a diffeomorphism, then

$$
\left(\psi_{*} X\right)(y)=D \psi\left(\psi^{-1}(y)\right) X\left(\psi^{-1}(y)\right)
$$

for $y \in V$. The vector field $\psi_{*} X$ is locally Lipschitz, $C^{n}$ or smooth if $X$ has the respective property. Notice that in our case the push forward of $A$ causes difficulties since it is a densely defined, non-continuous, non-linear vector field.

Theorem 2. Let $\psi: U \rightarrow V$ be a smooth diffeomorphism of open sets $U, V \subset H$ and assume that $\psi(U \cap \mathcal{D}(A))=V \cap \mathcal{D}(A)$. Furthermore we assume

$$
\psi_{*} A=A+\zeta,
$$

where $\zeta: V \rightarrow H$ is a locally Lipschitz vector field, on $V \cap \mathcal{D}(A)$, i.e.

$$
D \psi\left(\psi^{-1}(y)\right) A \psi^{-1}(y)=A y+\zeta(y)
$$

for $y \in V \cap \mathcal{D}(A)$. Then for any mild, continuous solution $\left(\phi_{t}\right)_{t \geq 0}$ of 2.1 with associated stopping time $\tau$ and initial value $\phi^{*}$, the continuous stochastic process $\left(\psi\left(\phi_{t}\right)\right)_{t \geq 0}$ is a mild, continuous solution of

$$
d \xi_{t}=\left(A \xi_{t}+\left(\psi_{*} \alpha\right)\left(\xi_{t}\right)+\zeta\left(\xi_{t}\right)+\chi\left(\xi_{t}\right) d t+\sum_{i=1}^{d}\left(\psi_{*} \beta_{i}\right)\left(\xi_{t}\right) d W_{t}^{i}\right.
$$

with initial value $\psi\left(\phi^{*}\right)$ (up to the same stopping time).

We denote the Ito correction term by $\chi$, i.e.

$$
\chi(y)=\sum_{i=1}^{d} D^{2} \psi\left(\psi^{-1}(y)\right)\left(\beta _ { i } \left(\psi^{-1}(y), \beta_{i}\left(\psi^{-1}(y)\right)\right.\right.
$$

for $y \in V$.

Remark 1. Notice that the difficulty of the theorem arises from the fact that mild, continuous solutions are not Ito processes in general. Therefore we cannot directly apply Ito's formula. The Theorem also holds under the assumption that $\psi(U \cap$ $\mathcal{D}(A))=V \cap \mathcal{D}(B)$ for generator $B: \mathcal{D}(B) \rightarrow H$ and $\psi_{*} A=B+\zeta$ on $V \cap \mathcal{D}(B)$. 
Proof. We assume first that $\alpha, \beta_{1}, \ldots, \beta_{d}$ are Lipschitz from $H$ to $\mathcal{D}(A)$, then we can apply the conclusions of Proposition 2. Given a mild, continuous solution $\left(\phi_{t}\right)_{t \geq 0}$ with initial value $\phi^{*} \in \mathcal{D}(A) \cap U$, this solution is a strong solution and

$$
\phi_{t}=\phi^{*}+\int_{0}^{t} A \phi_{s} d s+\int_{0}^{t} \alpha\left(\phi_{s}\right) d s+\sum_{i=1}^{d} \int_{0}^{t} \beta_{i}\left(\phi_{s}\right) d W_{s}^{i}
$$

holds for $0 \leq t \leq \tau$. By Ito's formula Theorem 1 we obtain

$$
\begin{aligned}
\psi\left(\phi_{t}\right) & =\psi\left(\phi^{*}\right)+\int_{0}^{t}\left(\psi_{*} A\right)\left(\psi\left(\phi_{s}\right)\right) d s+\int_{0}^{t} \psi_{*} \alpha\left(\psi\left(\phi_{s}\right)\right) d s+ \\
& +\int_{0}^{t} \chi\left(\psi\left(\phi_{s}\right) d s+\sum_{i=1}^{d} \int_{0}^{t} \psi_{*} \beta_{i}\left(\psi\left(\phi_{s}\right)\right) d W_{s}^{i}\right. \\
& =\psi\left(\phi^{*}\right)+\int_{0}^{t} A\left(\psi\left(\phi_{s}\right)\right) d s+\int_{0}^{t} \zeta\left(\psi\left(\phi_{s}\right)\right) d s+\int_{0}^{t} \psi_{*} \alpha\left(\psi\left(\phi_{s}\right)\right) d s+ \\
& +\int_{0}^{t} \chi\left(\psi\left(\phi_{s}\right) d s+\sum_{i=1}^{d} \int_{0}^{t} \psi_{*} \beta_{i}\left(\psi\left(\phi_{s}\right)\right) d W_{s}^{i} .\right.
\end{aligned}
$$

Since $\phi_{t} \in \mathcal{D}(A)$ we obtain the desired formulation by $\psi_{*} A=A+\xi$ on $V \cap \mathcal{D}(A)$. Every strong solution is a mild solution, hence

$$
\begin{aligned}
\psi\left(\phi_{t}\right) & =T_{t} \psi\left(\phi^{*}\right)+\int_{0}^{t} T_{t-s}(\zeta)\left(\psi\left(\phi_{s}\right)\right) d s+\int_{0}^{t} T_{t-s} \psi_{*} \alpha\left(\psi\left(\phi_{s}\right)\right) d s+ \\
& +\int_{0}^{t} T_{t-s} \chi\left(\psi\left(\phi_{s}\right) d s+\sum_{i=1}^{d} \int_{0}^{t} T_{t-s} \psi_{*} \beta_{i}\left(\psi\left(\phi_{s}\right)\right) d W_{s}^{i}\right.
\end{aligned}
$$

for $0 \leq t \leq \tau$. Given any initial value $\phi^{*} \in U$, we can find a sequence $\phi^{n} \rightarrow \phi^{*}$ with $\phi^{n} \in U \cap \mathcal{D}(A)$, a strictly positive stopping time $\tau$ and mild solutions $\left(\phi_{t}^{n}\right)_{t \geq 0}$ and $\left(\phi_{t}\right)_{t \geq 0}$ such that $E\left(\sup _{0 \leq t \leq \tau}\left\|\phi_{t}^{n}-\phi_{t}\right\|_{H}^{2}\right) \rightarrow 0$. The mild solutions $\phi^{n}$ are strong solutions and by equation 2.2 , which is valid for each $\phi^{n}$, we can pass to the limit in probability, which yields the result

$$
\begin{aligned}
\psi\left(\phi_{t}\right) & =T_{t} \psi\left(\phi^{*}\right)+\int_{0}^{t} T_{t-s}(\zeta)\left(\psi\left(\phi_{s}\right)\right) d s+\int_{0}^{t} T_{t-s} \psi_{*} \alpha\left(\psi\left(\phi_{s}\right)\right) d s+ \\
& +\int_{0}^{t} T_{t-s} \chi\left(\psi\left(\phi_{s}\right) d s+\sum_{i=1}^{d} \int_{0}^{t} T_{t-s} \psi_{*} \beta_{i}\left(\psi\left(\phi_{s}\right)\right) d W_{s}^{i}\right.
\end{aligned}
$$

for $0 \leq t \leq \tau$. This holds for every stopping time which is associated to $\phi$ by uniqueness.

For the general case we change the vector fields in question by the resolvent: by semigroup theory we know that $n R(A, n) x \rightarrow x$ as $n \rightarrow \infty$ for all $x \in H$, where $A$ denotes the generator of $T_{t}$ and $R(A, \lambda)$ its resolvent. Therefore the vector fields $\alpha^{n}:=n R(A, n) \alpha$ and $\beta_{i}^{n}:=n R(A, n) \beta_{i}$ for $i=1, \ldots, d$ are well defined vector fields which take values in $\mathcal{D}(A)$ and which converge pointwise to $\alpha$ and $\beta_{i}$. For these vector fields the conclusions of the first part hold, i.e. for any mild, continuous solution $\phi^{n}$ with respect to $A+\alpha^{n}, \beta_{1}^{n}, \ldots, \beta_{d}^{n}$ the transformed process $\left(\psi\left(\phi_{t}^{n}\right)\right)_{t \geq 0}$ 
is a mild, continuous solution with respect to $A+\psi_{*} \alpha^{n}+\chi+\zeta, \psi_{*} \beta_{1}^{n}, \ldots, \psi_{*} \beta_{d}^{n}$ :

$$
\begin{aligned}
\psi\left(\phi_{t}^{n}\right) & =T_{t} \psi\left(\phi^{*}\right)+\int_{0}^{t} T_{t-s}(\zeta)\left(\psi\left(\phi_{s}^{n}\right)\right) d s+\int_{0}^{t} T_{t-s} \psi_{*} \alpha^{n}\left(\psi\left(\phi_{s}^{n}\right)\right) d s+ \\
& +\int_{0}^{t} T_{t-s} \chi\left(\psi\left(\phi_{s}^{n}\right)\right) d s+\sum_{i=1}^{d} \int_{0}^{t} T_{t-s} \psi_{*} \beta_{i}^{n}\left(\psi\left(\phi_{s}^{n}\right)\right) d W_{s}^{i} .
\end{aligned}
$$

By Gronwall's lemma we obtain that $E\left(\sup _{0 \leq t \leq \tau}\left\|\phi_{t}^{n}-\phi_{t}\right\|_{H}^{2}\right) \rightarrow 0$, see [4], which allows to pass to the desired limit.

Remark 2. Notice that the assumption $\psi_{*} A=A+\zeta$ is crucial for the fact, that the transformed mild solution is indeed a mild solutions of an equation of type 2.1.

\section{Applications to Energy markets}

For the term structure problem we are investigating equations of the type

$$
d \phi_{t}=\left(\frac{d}{d x} \phi_{t}-\frac{1}{2} \sum_{i=1}^{d} \sigma_{i}^{2}\left(t, \phi_{t}\right)+\gamma\left(t, \phi_{t}\right)\right) d t+\sum_{i=1}^{d} \sigma_{i}\left(t, \phi_{t}\right) d W_{t}^{i}
$$

on a Hilbert space of term structures $\phi \in H \subset C\left(\mathbb{R}_{\geq 0}, \mathbb{R}\right)$. These Hilbert spaces are characterized by the following axioms (compare and find details in [5]):

H1: The Hilbert space $H$ is a Hilbert space of continuous functions and point evaluations are continuous linear functionals.

H2: The shift semigroup $\left(S_{t} f\right)(x):=f(x+t)$ is a strongly continuous semigroup on $H$ with generator $\frac{d}{d x}$.

H3: There is a closed subspace $H_{0} \subset H$ of finite codimension such that the multiplication $(f, g) \mapsto f g$ is a well-defined bounded bilinear map from $H_{0} \times H_{0} \rightarrow H$.

H4: Let $g \in C^{\infty}\left(\mathbb{R}_{>0} \times \mathbb{R}, \mathbb{R}\right)$ be given, such that there is $\delta>0$ with $g(x, y)=$ $y$ for $x \geq \delta$. Then $f \mapsto \psi_{g}(f)(x):=g(x, f(x))$ for $x \geq 0$ is smooth on the Hilbert space $H$.

Existence of such Hilbert spaces can be proved by the methods of [5], they appear to be Sobolev-type spaces on $\mathbb{R}_{>0}$ with some measures punishing high values of certain derivatives for large times to maturity.

Lemma 1. Given a Hilbert space $H$ satisfying (H1)-(H4), then

$$
\begin{gathered}
\left(D \psi_{g}(f) \cdot h\right)(x)=\frac{\partial}{\partial y} g(x, f(x)) h(x) \\
\left(D^{2} \psi_{g}(f) \cdot\left(h_{1}, h_{2}\right)\right)(x)=\frac{\partial^{2}}{\partial y^{2}} g(x, f(x)) h_{1}(x) h_{2}(x) .
\end{gathered}
$$

Proof. We have to calculate the directional derivative, which can be done under point evaluations, since point evaluations are continuous linear functionals and for $l \in H^{\prime}$ and $\psi \in C^{\infty}(H, H)$ we have $\frac{d}{d t}(l \circ \psi)(f+t h)=l\left(\frac{d}{d t} \psi(f+t h)\right)$. The point evaluations are point separating, so we can characterize the derivative completely,

$$
\left.\frac{d}{d t}\right|_{t=0} \psi_{g}(f+t h)(x)=\frac{\partial}{\partial y} g(x, f(x)) h(x) .
$$

The second derivative is calculated as derivative of $D \psi: H \rightarrow L(H, H)$, which also can be calculated via two fold evaluations, namely at $h_{1} \in H$ and then at $x \geq 0$. For the analysis involved see [7]. 
Assume (H1)-(H4) to be in force. Let $g \in C^{\infty}\left(\mathbb{R}_{\geq 0} \times \mathbb{R}, \mathbb{R}\right)$ be given such that there is $\delta>0$ with $g(x, y)=y$ for $x \geq \delta$ and derivative $\frac{\partial}{\partial y} g(x, y)$ bounded away from zero for all $x \geq 0$ and $y \in \mathbb{R}$. Then we can define $\psi_{g}(f)(x):=g(x, f(x))$ for $x \geq 0$, which is assumed to be smooth on the given Hilbert space $H$ by (H4). This transformation represents a change of the term structure on the interval $[0, \delta]$.

Theorem 3. Let $U \subset H$ be an open subset of a Hilbert space satisfying (H1)-(H4) and assume that $\psi_{g}: U \rightarrow V$ is a diffeomorphism. Let $\left(\phi_{t}\right)_{t \geq 0}$ be a mild solution of equation 3.1 up to some stopping time $\tau>0$, then the stochastic process $\left(\psi_{g}\left(\phi_{t}\right)\right)_{t \geq 0}$ is a mild, continuous solution of the Stochastic Differential Equation

$$
d \eta_{t}=\left(\frac{d}{d x} \eta_{t}-\frac{1}{2} \sum_{i=1}^{d} \widetilde{\sigma}_{i}^{2}\left(\eta_{t}\right)+\gamma\left(\eta_{t}\right) d t+\sum_{i=1}^{d} \widetilde{\sigma}_{i}\left(\eta_{t}\right) d W_{t}^{i}\right.
$$

with initial value $\psi_{g}\left(\phi^{*}\right)$ up to $\tau$. The perturbed volatility vector fields are given through

$$
\begin{aligned}
\widetilde{\sigma}_{i}(f) & =D \psi_{g}\left(\psi_{g}^{-1}(f)\right) \sigma_{i}\left(\psi_{g}^{-1}(f)\right) \\
& =\left(\psi_{g}\right)_{*} \sigma_{i}
\end{aligned}
$$

and the perturbation of the drift is given through

$$
\begin{aligned}
\gamma(f) & =-\psi_{\frac{\partial}{\partial x} g}\left(\psi_{g}^{-1}(f)\right)+\frac{1}{2} \sum_{i=1}^{d}\left(\left(\psi_{g}\right)_{*} \sigma_{i}\right)^{2}-\frac{1}{2} \sum_{i=1}^{d}\left(\psi_{g}\right)_{*} \sigma_{i}^{2}(f) \\
& +\frac{1}{2} \sum_{i=1}^{d} D^{2} \psi_{g}\left(\psi_{g}^{-1}(f)\right)\left(\sigma_{i}\left(\psi_{g}^{-1}(f)\right), \sigma_{i}\left(\psi_{g}^{-1}(f)\right)\right),
\end{aligned}
$$

where we assume that there is $h \in C^{\infty}\left(\mathbb{R}_{\geq 0} \times \mathbb{R}, \mathbb{R}\right)$ with $\psi_{g}^{-1}=\psi_{h}$.

Remark 3. Notice that the point evaluations for $x \geq \delta$ yield $\gamma(f)(x)=0$ and $\widetilde{\sigma}_{i}(f)(x)=\sigma_{i}(f)(x)$. Beyond the critical time the vector fields remain unchanged.

Proof. Apply Theorem 2, since

$$
\begin{aligned}
\left(\left(\psi_{g}\right)_{*} \frac{d}{d x}\right) \psi_{g}(h)(x) & =\left(D \psi_{g}(h) \cdot \frac{d}{d x} h\right)(x)=\frac{\partial}{\partial y} g(x, f(x)) \frac{d}{d x} h(x) \\
& =\frac{d}{d x} g(x, h(x))-\frac{\partial}{\partial x} g(x, h(x)) \\
& =\frac{d}{d x} \psi_{g}(h)(x)-\psi_{\frac{\partial}{\partial x} g}(h)(x),
\end{aligned}
$$

hence

$$
\left(\left(\psi_{g}\right)_{*} \frac{d}{d x}\right) f=\frac{d}{d x} f-\psi_{\frac{\partial}{\partial x} g}\left(\psi_{g}^{-1}(f)\right)
$$

holds for $f \in \mathcal{D}\left(\frac{d}{d x}\right)$ and $f \mapsto \psi_{\frac{\partial}{\partial x}} h\left(\psi_{g}^{-1}(f)\right)$ is a locally Lipschitz vector field.

We want to construct flexible and tractable stochastic models, which incorporate an affine behaviour for times to maturity $x \geq \delta$ and some irregular behaviour for small times to maturity. The method proposed by Theorem 2 is to take a general affine model and to transform it by an adequate choice of $\psi_{g}$.

We choose a smooth, monotonic function $f_{\alpha}: \mathbb{R} \rightarrow \mathbb{R}$, depending on a real parameter $\alpha \neq 0$, such that

$$
f_{\alpha}(y)=(1+\alpha y)^{\frac{1}{\alpha}} \text { for } 1+\alpha y>\epsilon
$$


for some fixed $0<\epsilon<1$. Notice that $f_{\alpha}(y) \rightarrow \exp (y)$ as $\alpha \rightarrow 0$, therefore we define $f_{0}(y):=\exp (y)$. This yields $f \in C^{\infty}\left(\mathbb{R}^{2}, \mathbb{R}\right)$, since the extension holds for all $y$-derivatives, too. Next choose a smooth function $\alpha$ on $\mathbb{R}_{\geq 0}$ with compact support, $\alpha_{0} \neq 0$ and

Then we can define

$$
\alpha(x)=\left\{\begin{array}{c}
\alpha_{0} \text { for } x=0 \\
0 \text { for } x \geq \delta
\end{array}\right.
$$

$$
g(x, y):=\ln \left(f_{\alpha(x)}(y)\right),
$$

which is a smooth function $g \in C^{\infty}\left(\mathbb{R}_{\geq 0} \times \mathbb{R}, \mathbb{R}\right), g(x, y)=y$ for $x \geq \delta$ and $\frac{\partial}{\partial y} g(x, y)=\frac{1}{f_{\alpha(x)}(y)} \frac{\partial}{\partial y} f_{\alpha(x)}(y)>0$. The inverse $h$ (whose existence is needed in order to apply Theorem 3) can be calculated directly.

Henceforward we shall assume that the volatility vector fields depend on the term structure in the following way

$$
\sigma_{i}(\phi)=\eta_{i}\left(l_{1}(\phi), \ldots, l_{n}(\phi)\right)
$$

where $\eta_{i}: \mathbb{R}^{n} \rightarrow \mathcal{D}\left(\left(\frac{d}{d x}\right)^{\infty}\right)$ is smooth and $l_{i}: \mathcal{D}\left(\left(\frac{d}{d x}\right)^{\infty}\right) \rightarrow \mathbb{R}$ are bounded linear functionals. We assume furthermore the interpolation condition of [6], which asserts that for all $r \geq 0$ the map

$$
\phi \mapsto\left(l_{1}(\phi), \ldots, l_{n}(\phi), \ldots, l_{1}\left(\frac{d^{r}}{d x^{r}} \phi\right), \ldots, l_{n}\left(\frac{d^{r}}{d x^{r}} \phi\right)\right)
$$

is surjective. Inserting point evaluations for $l_{i}=e v_{x_{i}}$ with $0 \leq x_{1}<\cdots<x_{n}$ this translates to the fact, that for any prescribed values of derivatives up to order $r$ at the points $x_{i}$ there is a function $\phi \in \mathcal{D}\left(\left(\frac{d}{d x}\right)^{\infty}\right)$ taking precisely these values. In this case - by the assertions in [6] - the conclusion holds that the term structure evolution is affine (and that the factor processes involved are affine processes). Therefore we can assume $\sigma_{i}(\phi)=\sum_{j=1}^{m} k_{i j}(\phi) \lambda_{j}$ where $\lambda_{j} \in \mathcal{D}\left(\left(\frac{d}{d x}\right)^{\infty}\right)$ and $k_{i j}$ : $U \rightarrow \mathbb{R}$ smooth.

Theorem 4. Given 1-factor model on $U \subset H$, i.e. $d=1$ and $\sigma(\phi)=k(\phi) \lambda$, i.e. the solution process satisfies of the classical term structure equation

$$
d \phi_{t}=\left(\frac{d}{d x} \phi_{t}-\frac{1}{2} \sum_{i=1}^{d} \sigma_{i}^{2}\left(\phi_{t}\right)\right) d t+\sum_{i=1}^{d} \sigma_{i}\left(\phi_{t}\right) d W_{t}^{i}
$$

satisfies $\phi_{t}(x)=\lambda_{0}(t, x)+\lambda_{1}(x) Z_{t}$. Then a (generalized) stochastic evolution of future prices is given by the following formula for an arbitrary initial state $T \mapsto$ $\ln \left(f_{\alpha(T)}\left(\phi^{*}(T)\right)\right.$

$$
F(t, T)=f_{\alpha(T-t)}\left(\lambda_{0}(t, T-t)+\lambda_{1}(T-t) Z_{t}\right)
$$

in particular

$$
S_{t}=\left(\lambda_{0}(t, 0)+\alpha_{0} Z_{t}\right)^{\frac{1}{\alpha_{0}}}
$$

if $\left(Z_{t}\right)_{t \geq 0}$ is below the the level $\frac{\epsilon-1}{\alpha_{0}}$ for $t \geq 0$. Notice that for $Z_{0}=0$ and in the case, that $Z_{t}$ is a Gaussian Ornstein-Uhlenbeck process, this corresponds exactly to Martin Barlow's model with time-dependent coefficients.

Proof. We apply the methods of [6] to calculate the 1-factor models, i.e. stochastic evolutions of

$$
d \phi_{t}=\left(\frac{d}{d x} \phi_{t}-\frac{1}{2} \sum_{i=1}^{d} \sigma_{i}^{2}\left(\phi_{t}\right)\right) d t+\sum_{i=1}^{d} \sigma_{i}\left(\phi_{t}\right) d W_{t}^{i}
$$


on $U \subset H$, such that generic 2-dimensional realizations exist (time is the second dimension besides the stochastic factor). First we calculate the derivatives

$$
\begin{aligned}
D \sigma(f) \cdot h & =(D k(f) \cdot h) \lambda \\
D \sigma(f) \cdot \sigma(f) & =k(f) \lambda D k(f) \cdot \lambda=p(f) \lambda \\
\mu(f) & =\frac{d}{d x} f+\frac{1}{2} k(f)^{2} \lambda^{2}-\frac{1}{2} p(f) \lambda \\
D \mu(f) \cdot h & =\frac{d}{d x} h+k(f) \lambda^{2} D k(f) \cdot h-\frac{1}{2} \lambda D p(f) \cdot h,
\end{aligned}
$$

hence we can calculate the Lie bracket

$$
[\mu, \sigma](f)=k(f) \frac{d}{d x} \lambda+k(f)^{2} \lambda^{2} D k(f) \cdot \lambda-\frac{1}{2} \lambda k(f) D p(f) \cdot \lambda-(D k(f) \cdot \mu(f)) \lambda .
$$

We assume that $[\mu, \sigma](f) \in\langle\lambda\rangle$ for $f \in U$, since we aim for 2-dimensional realizations, and obtain therefore an equation of the type

$$
\frac{d}{d x} \lambda+q_{1}(f) \lambda^{2}+q_{2}(f) \lambda=0,
$$

which amounts to solve a Riccati equation for $\lambda$. We assume $\lambda(0)=1$, which is possible by rescaling $k$ and obtain the equation

$$
\frac{d}{d x} \lambda+a \lambda^{2}+b \lambda=0
$$

for the term structure direction. We can consequently write - up to a certain stopping time - the evolution of an initial value $\phi^{*}$ by

$$
\phi_{t}=\lambda_{0}(t)+\lambda Z_{t}
$$

with a stochastic factor process $\left(Z_{t}\right)_{t \geq 0}$ for $0 \leq t \leq \tau$. Here $\lambda_{0}(t)=F l_{t}^{\mu}\left(\phi^{*}\right)$. This solution is then transformed by $\psi_{g}$, which yields the result.

Remark 4. One can easily calculate the covariance structure of the future prices in the critical interval $0 \leq T-t \leq \delta$ by the explicit formula for $g$ and Theorem 2.

The question what is the meaning of $Q$ for $0 \leq x \leq \delta$ remains open. From the point of view of Girsanov's theorem one knows that there is a market price of risk, namely some predictable process $\left(\gamma_{1}, \ldots, \gamma_{d}\right): \Omega \times H \rightarrow \mathbb{R}^{d}$ such that the real world drift is given by

$$
\mu(\phi)=\frac{d}{d x} \phi-\frac{1}{2} \sum_{i=1}^{d} \sigma_{i}^{2}(\phi)+\sum_{i=1}^{d} \gamma_{i}(\phi) \sigma_{i}(\phi)+\gamma(\phi),
$$

which means the drift is changed even for $0 \leq x \leq \delta$. Nevertheless it might be useful to realize that this change can be neglected with respect to $\gamma$ at least near 0 , since $\gamma$ contains one term producing high values independent of the volatilities of the model, which are typically low. So $Q$ could be regarded as mixture between the physical measure for $\left(S_{t}\right)_{t \geq 0}$ and the martingale measure for the market. By no means $Q$ can be regarded as no arbitrage pricing measure for $\left(S_{t}\right)_{t \geq 0}$ !

Remark 5. One can do the above procedure also in cases, where there are given disjoint intervals $I_{j}=\left[a_{j}, b_{j}\left[\subset \mathbb{R}_{\geq 0}\right.\right.$ for $j=1, \ldots, n$ and where one assumes that

$$
(F(t, T))_{T-t \notin I_{j}}
$$

is a $Q$-martingale for $j=1, \ldots, n$. 


\section{REFERENCES}

[1] M. Barlow, A diffusion model for electricity pricing, Math. Finance 12, No.4, 287-298 (2002).

[2] T. Björk, C. Landén, On the term structure of futures and forward prices, Mathematical Finance - Bachelier Congress 2000, Springer Verlag Berlin, Springer Finance (2002).

[3] J. Hull, Options, futures, and other derivatives, Prentice-Hall International Editions. Upper Saddle River, NJ: Prentice Hall. xxi (2003).

[4] G. Da Prato, J. Zabczyk, Stochastic equations in infinite dimensions, Cambridge University Press, 1992.

[5] D. Filipović, Consistency problems for Heath-Jarrow-Morton interest rate models, SpringerVerlag, Berlin, 2001.

[6] D. Filipović, J. Teichmann, Existence of finite dimensional realizations for stochastic equations, J. Funct. Anal. 197, No.2, 398-432 (2003).

[7] Andreas Kriegl and Peter W. Michor, The convenient setting for global analysis, 'Surveys and Monographs 53', AMS, Providence, 1997.

Research Group of financial and actuarial mathematics E105, Institute of mathematical methods in economics, Technical University of Vienna, Wiedner Hauptstrasse 8-10, A-1040 Vienna, Austria

E-mail address: josef.teichmann@fam.tuwien.ac.at 